\title{
Epidemiological Analysis on 183 Cases of Congenital Syphilis
}

\author{
Hui $\mathrm{Wu}^{1, \mathrm{a}}$, Jing Cui ${ }^{1, \mathrm{~b}}$, Dan Dang ${ }^{1, \mathrm{c}}$, Dongxuan Wang ${ }^{2, \mathrm{~d}, *}$ \\ 1. Department of Neonatology, the First Hospital of Jilin University, Chang Chun, 130021, China \\ ${ }^{2}$ Department of Ultrasonic Diagnosis, The First Hospital of Jilin University, Changchun , China ,130021 \\ a wuhui97@126.com, b cuijing_1029@163.com, \\ c695344998@qq.com, d dongxuanwang@hotmail.com \\ * Corresponding author : Dongxuan Wang , Email: dongxuanwang@hotmail.com
}

\begin{abstract}
Purpose:To discuss the epidemiological characteristics of congenital syphilis. Methods: Retrospective analysed the 183 cases of congenital syphilis from Jan. 2002 to Jan. 2012 in our department, and the gestational age, sex, birth weight, delivery mode, age on admission, onset time, parents' occupations, medical history and treatment of mother's syphilis are analyzed. Results: In the past ten years, rate of congenital syphilis in total inpatients increased, $7.7 \%$ are latent syphilis, $92.3 \%$ are early congenital syphilis, and there is no late congenital syphilis. It is more common for babies of three months, $68.3 \%$ are neonates. $36.1 \%$ are diagnosed in prenatal period, and 3.3\% treated with full course of penicillin. Conclusion: Incidence of congenital syphilis increased year by year, the detectable rate of syphilis in prenatal period and the rate of full course treatment are low, most congenital syphilis are within 3 months after birth, especially in neonatal period.
\end{abstract}

Keywords. Congenital; Syphilis; Neonates; Epidemiology

Congenital syphilis (CS), also termed as prenatal syphilis, is an infectious disease caused by treponema pallidum (TP) in the body of the mother passing through the placenta into the blood circulation of the fetus, which does severe harm to the health of fetus and neonate. In recent year, the incidence of syphilis is on the increase year by year around the world [1,2], and CS is increasing accordingly, and the situation is the same in China [3-5] and should arouse wide concern. Based on the existing literature concerning CS, the statistical analysis is conducted on the clinical data about CS cases of our department in the recent ten years from Jan. 2002 to Jan. 2012 in this research, aiming to discuss the epidemiological characteristics of CS incidence in this center.

\section{$1 \quad$ Materials and methods}

\subsection{Research object}

There are 183 cases of CS sick children who met the diagnostic criteria [6], received and cured in neonatal department of the First Hospital of Jilin University from Jan. 2002 to Jan. 2012, among which, there are 125 cases of neonates, 55 cases of babies from 29 days to 3 months, 3 cases of babies from 3 months to 2 years.

\subsection{Research method}

The data of 183 clinical cases of CS sick children during the past ten tears is analyzed retrospectively, and its epidemiological characteristics are discussed.

Basic information . The gestational age, sex, birth weight, delivery mode, the age on admission and the age in days of onset of disease of sick children are included.

Basic information about family . The occupations of parents and the medical history and treatment of mother's syphilis

\section{Result}

\subsection{Basic information about sick children}

According to the study of our center, it can be seen that from Jan. 2002 to Jan. 2012, the rates of CS sick children of our department in the total sick children in hospital of the corresponding period each tear are $0.16 \%$ (1case), $0.41 \%$ (4 cases), $0.34 \%$ (5 cases), $0.58 \%$ (10 cases), $0.62 \%$ (12 cases), $0.79 \%$ (19 cases), $0.53 \%$ (17 cases), $1.04 \%$ (37 cases), $0.76 \%$ (28 cases) and 1.52\% (50 cases), 
which are on the increase year by year. The incidence is still high although the cases in 2008 and 2010 decreased slightly. See fig.1.

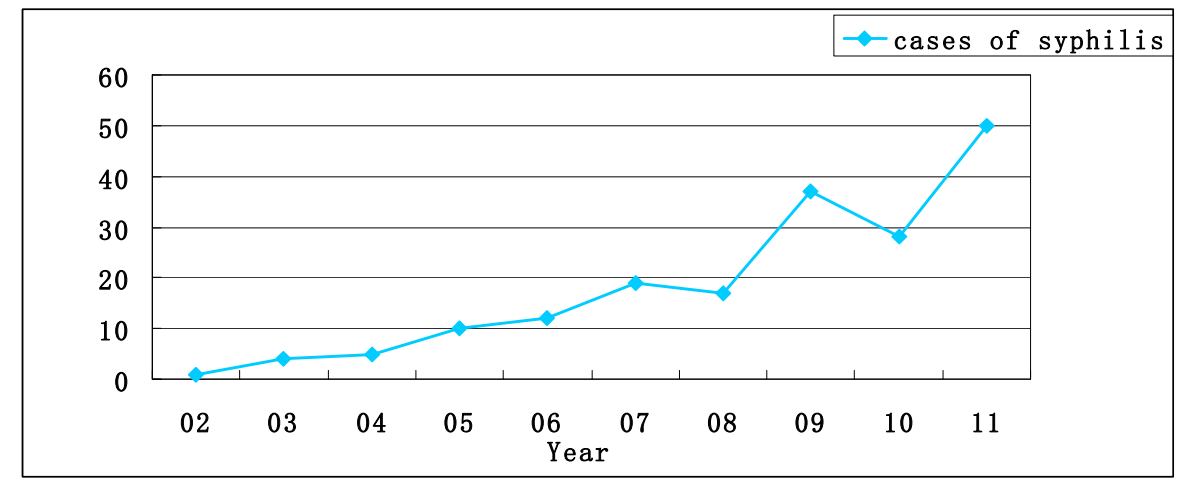

Fig. 1. The cases of congenital syphilis in hospital from Jan. 2002 to Jan. 2012

Gestational age. The gestational age is between 29 weeks and 42 weeks ${ }^{5 / 7}$, among which $32.8 \%$ (60/183) are premature infants, and the rates in that of the corresponding period are $0,0,0,0.38 \%$ (1/262), $0.35 \%$ (1/282), $0.64 \%$ (4/626), $0.54 \%$ (5/921), 1.5\% (16/1072), 1.1\% (13/1194) and $1.3 \%$ (20/1549); $67.2 \%(123 / 183)$ are term infants, and the rates in that of the corresponding period are $0.16 \%$ (1/611), $0.41 \%$ (4/985), $0.34 \%$ (5/1484), $0.63 \%$ (9/1432), $0.66 \%$ (11/1655), $0.84 \%$ (15/1779), $0.52 \%$ (12/2295), $0.85 \%$ (21/2485), $0.61 \%$ (15/2467) and $1.23 \%$ (30/2447). See fig.2.

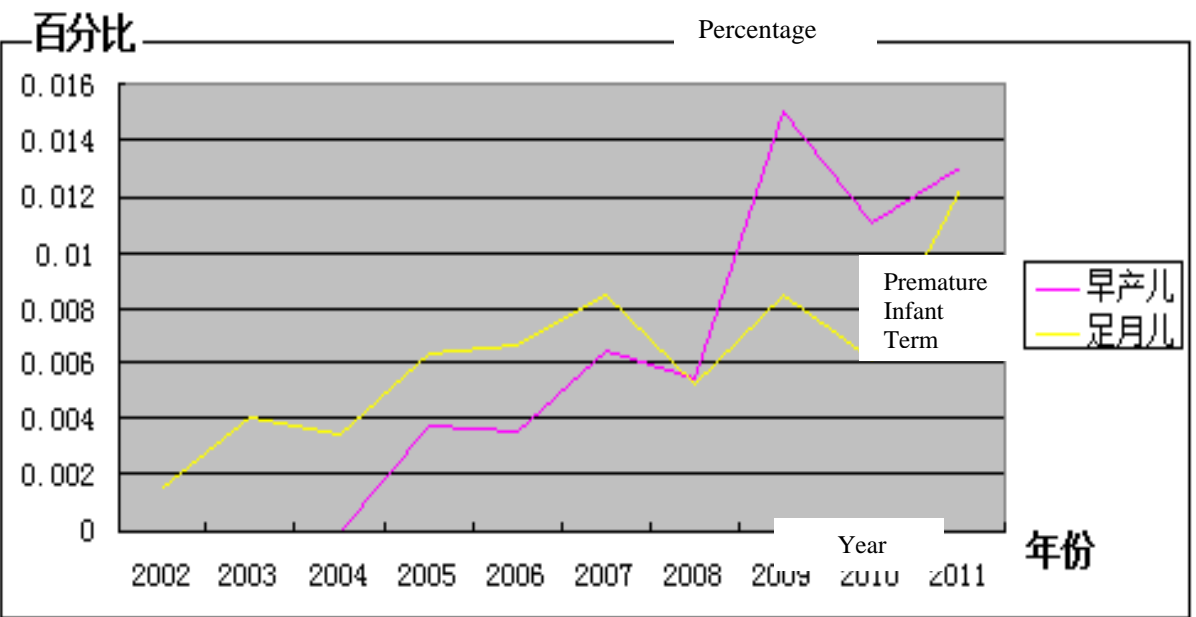

Fig. 2. The distribution of gestational age of sick children suffered from congenital syphilis in our center

Sex . In our center, 56.8\% (104/183) sick children are boys, while $41.2 \%(79 / 183)$ are girls, the Male/Female ratio is about 1.32: 1 .

Birth weight. It waves between $1.03 \mathrm{~kg}$ and $5.42 \mathrm{~kg}$ (M: $2.78 \mathrm{~kg}), 30.6 \%(56 / 183)$ are low birth weight infants, $3.82 \%(7 / 183)$ are very low birth weight infants, and $65.6 \%(120 / 183)$ are normal weight infants.
Delivery mode. Among which, 44.8\% (82/183) are born through vaginal delivery, 55.2\% (101/183) are through cesarean delivery.

The age on admission. Among the 183 cases of sick children, the minimum age on admission is 0.5 hour after birth, the maximum age is 5 months after birth. There are no sick children of syphilis more than 2 years in record, among which, $68.3 \%(125 / 183)$ are neonates, 30.1\% (55/183) are babies within 3 months after birth; $1.6 \%$ 
(3/183) are babies more than 3 months after birth. The age composition is seen in fig.3.

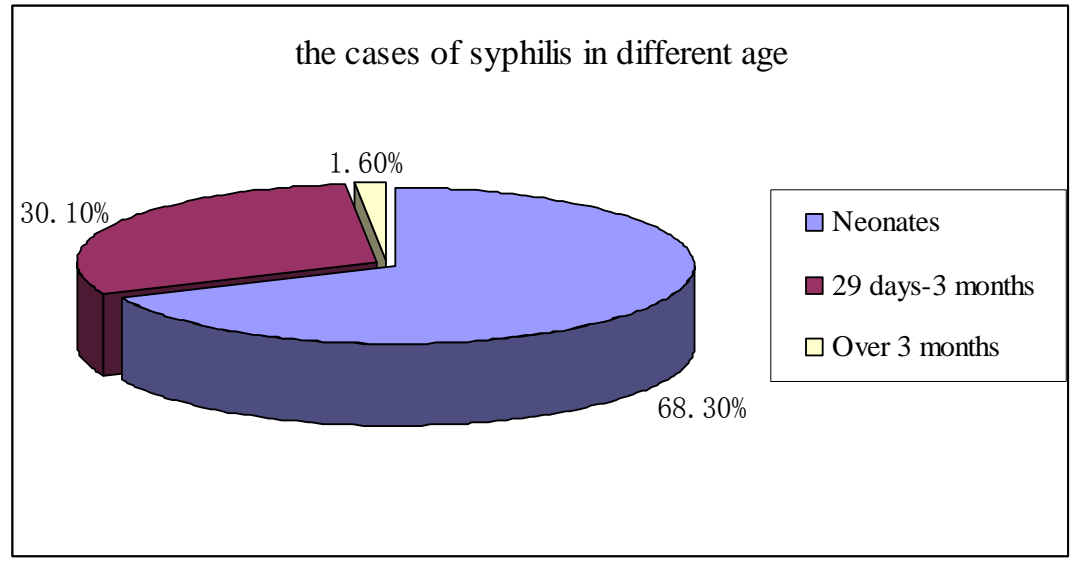

Fig. 3. The cases of syphilis of different age groups

Age in days of onset of disease. Among the 183 cases of sick children, there can be found no clinical symptoms or signs on $7.7 \%(14 / 183)$ of them, and thus they are latent syphilis patients, $92.3 \%(169 / 183)$ of sick children are in hospital because of various clinical symptoms, who are early CS patients, and there is no late CS. Among the 169 cases of sick children with early CS, 66.1\% (121/183) are sick children in neonatal period, the rates of sick children with emergent symptom within one day after birth, at 2 days to 2 weeks, 15 days to 4 weeks are $32.5 \%$ (55/169), $18.9 \%$ (32/169), 14.2\% (24/169), respectively. The rate of syphilis sick children with emergent clinical symptoms at 29 days to 3 months and after 3 months are 32.5\% (55/169) and 1.64\%(3/169). The age distribution is seen as fig.4.

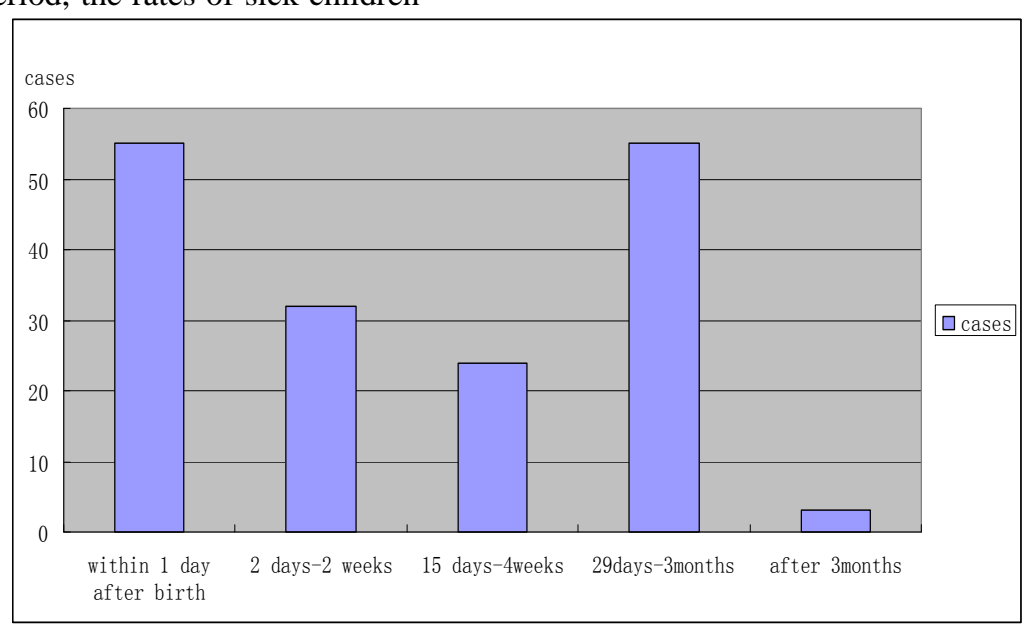

Fig. 4. The age distribution of early congenital syphilis with symptoms

\subsection{Basic information of the family}

Among the 183 cases of sick children diagnosed with CS, the rate of the mothers diagnosed with syphilis in prenatal period is $36.1 \%$ (66/183), which includes the 41 cases diagnosed in pregnancy screening and the 25 cases with medical history of syphilis, among which, 9.3\% (17/183) are treated with penicillin antisyphilitic treatment before the delivery, and there are 6 cases with 4 weeks of full course treatment, which accounting for $3.3 \%$, there are 1 case for course of treatment of 3 weeks, 2 weeks, 10 days and 3 days, respectively. Meanwhile, there are 7 cases whose treatment conditions are unknown. Among those sick children whose mothers were treated with penicillin before the delivery, there are 2 cases $(11.8 \%)$ of premature, 6 cases (35.3\%) are low birth weight infants, and there is no very low birth weight infant or developmental malformation. When seeing the doctor, the change of sick 
children's RPR titer is from 1: 4 to 1: 128 (M: 1: 24.9); among the rest 49 cases of sick children whose mothers are diagnosed with syphilis before the delivery without penicillin treatment, 7 cases $(14.3 \%)$ are premature infants, 17 cases are $(34.7 \%)$ low birth weight infants, 2 cases $(4.1 \%)$ are very low birth weight infants, the change of RPR titer when seeing the doctor is from 1: 4 to 1: 512 (M: 41.3). Since 2011, the occupations of sick children's parents are recorded for neonatal cases, there are 34 cases of syphilis sick children with records of parents' occupations among the 50 cases in the same year, among which, $32.4 \%(11 / 34)$ are peasants, $29.4 \%(10 / 34)$ are the unemployed, $17.6 \%(6 / 34)$ are drivers, $11.8 \%(4 / 34)$ are individual households, $8.8 \%$ (3/34) are technicians.

\section{Discussion}

According to the statistic analysis of confirmed CS sick children in our department in the past ten years from Jan. 2002 to Jan. 2012, the incidence of CS is on the increase year by year. Among the 183 cases of CS sick children in this material, only 66 cases of sick children are diagnosed with syphilis in prenatal period, among which, only 17 cases are treated with penicillin in prenatal period, and only 6 cases are treated with full course, accounting for $3.3 \%$. As to the mothers of sick children suffering from congenital syphilis, $96.6 \%$ are syphilis infected persons without treatment or lack of full course of treatment. It can be concluded that the screening rate of syphilis for women in gestation period is low, and so is that of patients treated with full course of penicillin. This is one of major causes of the year-to-year increase of the incidence of congenital syphilis in recent years. Meanwhile, the case that although the pregnant woman has been treated with full course of penicillin, there is still possibility for the neonate to be ill should be paid attention to, this may be related to the time of treatment. It can also be seen from this material that compared with pregnant women without penicillin treatment in prenatal period, the occurrence rates of premature delivery, low birth weight and RPR titer for neonates are lower than those who have been treated with penicillin. It is also reported in some related records that compared with pregnant women without penicillin treatment progestational stage and women treated in pregnancy, the RPR positive rate for neonates whose mothers had been treated regularly before pregnancy is obviously lower. And the later of the treatment in pregnancy, the higher the incidence of CS and the severer the damages in multisystem are [7]. According to the statistics, the infection rate of fetus of pregnant women suffering from primary syphilis without treatment reaches up $70 \%$ to $100 \%$, and that of secondary and tertiary syphilis are $90 \%$ and 30\% [8], respectively. The occur- rence risk of bad outcome for women afflicted with syphilis in gestation period and those who have been treated is still as 2.5 times $[9,10]$ high as that of pregnant women without syphilis. Apart from treatment before pregnancy and in gestation period, the incidence of CS is also positive correlation with course of disease of syphilis in gestation period. During gestation period, the organism of woman is in immunosuppressive state, $90 \%$ pregnant women are suffered from latent syphilis without specific clinical symptoms, it can only be found through prenatal examination, pregnancy screening or prenatal syphilis serological examination [11]. Since our country has canceled compulsory antenatal care, and also, there are no specific manifestations for syphilis in pregnancy screening, latent syphilis can be easily neglected by most doctors. The delay of treatment opportunity can cause the increase of the amount of fetus infected with syphilis. The TP infection of fetus can occur at any time during gestation period, which will lead to abortion, stillbirth, premature delivery and other developmental malformation [12, 13]. Therefore, regular antenatal care and syphilis serological examination in gestation period should be strongly advocated. Once diagnosed with syphilis infected person, early and full course of treatment are needed to reduce congenital syphilis and complication. Although pregnancy screening is taken as conventional screening in some hospitals, the detection for women of mobile gestation age still remain blind zone. In addition, the occupations of the 34 cases of sick children's parents are recorded in this study, among which, 91.2\% (31/34) is peasant, individual household, the unemployed and driver. They are lack of health knowledge and pregnancy screening, thus, the propaganda work of prevention and treatment knowledge about venereal disease should be strengthened from now on, especially the pregnancy screening work for syphilis high-risk groups such as people in rural area, individual households, the unemployed, drivers and the like, so as to reduce the incidence of congenital syphilis,.

Apart from the 17 cases of latent syphilis, 92.3\% (169 cases) of the 183 cases in this group are early CS, it can be seen that early CS is more common in clinic, babies within 3 months after birth are the main sufferers, especially in neonatal period. Infection with TP in gestation period can cause abortion, stillbirth, premature delivery and low birth weight. According to relevant data, the incidence for premature infant with intrauterine infection of syphilis reaches up to $20 \%-50 \%$ [14]. As to the 183 cases of sick children in this group, among which, 60 cases are premature infants (32.8\%), 56 cases are low birth weight infants (30.6\%), 7 cases are very low birth weight infants (3.82\%), which is consistent with that reported in document. In recent 10 years, apart from the CS sick children from 2002 to 2004 are all not premature infants, the rate of premature infants of CS sick children in that of the 
corresponding period is on the increase year by year in the rest seven years. It can be seen that syphilis is also one cause of the year-to-year increase of premature infant. This calls upon us to do pregnancy screening of syphilis work actively to achieve early discovery and early treatment, aiming to reduce the incidence of CS and complication as much as possible and enhance life quality of neonates.

\section{References}

1. Centers for Disease Control and Prevention:The National Plan to Eliminate Syphilis from the United States. Atlanta . GA : U . S . Department of Health and Human Services . http : // WWW. cdc. gov/stopsyphilis/SEEPlan2006. Pdf.( 2006)

2. Sexually Transmitted Disease Surveillance.http: // www . ode. gov /std/stats08/ figures/43. htm, http: // www. nestde. ors/yqjc—detail/ epidemiology/ epidemiology_023. ppt.( 2008).

3. Oseph, D.T, Xiang, S.C., Rosanna, W. : Peeling. Syphilis and Social Upheaval in China. The New England Journal of Medicine.362,1658-1661. (2010)

4. China Disease Control Center statistics, Http: //www.ncstdc.org/yqjc- detail/ work/2009/work_030.pdf. (2009)

5. Wan, C.M.: Current Epidemiology of congenital syphilis. Journal of Applied Clinical Pediatrics. 21,1521-1522.( 2006)

6. Han, Z.J., Huang, D.M.,Guan, X.J.: practical neonatology. Beijing: People's Medical Publishing House, PP. 359 - 362. (2003)

7. Yuan, L.W. : Research progress of pregnancy syphilis infection and PMTCT . Medical Reviews. 3767-3769. (2009)

8. Sanchez, P.J., Gutman, L.T.:Textbook of pediatric infectious diseases. Philadelphia: Sauders .PP.1724-1741. (2004)

9. Lumhiganon, P., Piaggio, G., Villar, J.: The epidemiology of syphilis in pregnancy. Int. J. STD. AIDS. 13,486- 494.(2002)

10. World Health Organization. The global elimination of congenital syphilis : rationale and strategy for action. World Health Organization,( 2007).

11. Genc, M., Ledger, W.J. :Syphilis in pregnancy. Sex Transm Infect. 76, 73-79. (2000)

12. Zhang, Y. :Sexually transmitted disease infection during pregnany and mother to newborn infant transmission: A research progress. Chinese Journal of Nosocomiology. 12, 318-320. (2002)

13. Yang, F, Zhang, R., Zhou, H.: Diagnosis of neonatal congenital syphilis with treponema pallidum IgM
Western blot. Chinese Journal of Dermatology. 37, 326 - 327.( 2004)

14. Azimi, P.: Syphilis (Treponema pallidam). In: Behrman RE, Kliegman RM, Jenson HB. Nelson textbook of pediat rics . 17thed. Philadelphia: Saunders, PP.978- 979 (2004) 